\title{
Unidad didáctica para promover oralidad y escritura desde la historia local
}

\section{Didactic unit to promote orality and writing from local history}

Olga Mariela González López*

Fecha de recepción: 23 de noviembre del 2017

Fecha de aprobación: 27 de noviembre del 2017

Artículo de Investigación Científica y Tecnológica

\section{Resumen}

Este proyecto de investigación, tiene como objetivo elaborar una unidad didáctica para estudiantes de cuarto y quintodeprimaria, conelfinde mejorarlas competencias comunicativas, haciendo énfasis en oralidad y escritura a través del reconocimiento de la historia local de la sede Gaunza Abajo en Guateque (Boyacá). La propuesta está enmarcada bajo el paradigma socio-critico, enfoque de Investigación Cualitativa y el diseño Investigación Acción, está planteada en cuatro etapas, iniciando con un diagnóstico para determinar el nivel de desarrollo que tienen los estudiantes, luego diseño y elaboración de la unidad didáctica basada en referentes de calidad emanados por el Ministerio de Educación Nacional (MEN, 2010), después la aplicación, ejecución y análisis; por último, la socialización a través de la emisora. La unidad didáctica permitió que los niños fueran artífices del aprendizaje en interacción con el contexto. De este modo, se evidencia avance de los estudiantes en el proceso oral-escritor.

Palabras clave: competenciascomunicativas, historia local, escuela nueva, educación.
*Institución Educativa Técnica Enrique Olaya Herrera Sede Gaunza Abajo - Boyacá Colombia maris2974@hotmail.com

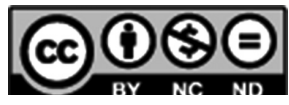




\section{Abstract}

This research project aims to develop a didactic unit for fourth and fifth grade students, in order to improve communication skills, emphasizing orality and writing through the recognition of the local history of the "Gaunza Abajo" school branch in Guateque (Boyacá). The proposal is framed under the socio-critical paradigm, Qualitative Research approach and Action Research design, proposed in four stages, starting with a diagnosis to determine the level of development that students have, then design and development of the teaching unit based on quality referents emanating from the Ministry of National Education (MEN, 2010), after the application, execution and analysis; finally, socialization through a radio station. The didactic unit allowed children to be the architects of learning in interaction with the context. In this way, students' progress is evident in the oral-writer process.

Keywords: communication skills, local history, new school, education. 


\section{Introducción}

La comunicación es fundamental en todas las áreas, disciplinas y procesos de la vida tanto individual como colectiva. Constituye uno de los grandes pilares del desarrollo personal, intelectual, familiar, social, emocional y cultural. Cuando comienza a fallar la comunicación, empiezan a debilitarse también los procesos de interacción con los demás. Ahí la importancia de fortalecer las competencias comunicativas, ya que si entre los adultos se precian dificultades de este orden, entre los niños son aún más marcados los inconvenientes al momento de comunicarse bien sea en forma oral o escrita.

En la Institución Educativa Técnica Enrique Olaya Herrera, sede Gaunza Abajo del municipio de Guateque, se es consciente de la importancia de fortalecer las competencias comunicativas, ya que en los resultados obtenidos de las pruebas saber 2015 la Institución se encuentra débil en el componente comunicativo escritor $\mathrm{y}$, por otra parte, las pruebas diagnósticas internas en el aula evidencian que los estudiantes presentan, dificultad para escribir con cohesión y coherencia; en cuanto a las pruebas de oralidad, se encontró que les cuesta trabajo expresarse, argumentar o participar en eventos culturales.

Es importante aclarar que, el problema no solamente está en la evaluación, sino que también se deben tener en cuenta la contextualización de los estudiantes y el currículo. Cada estudiante es un mundo diferente y, así como la escuela está encargada de formar personas capaces de asumir los diferentes retos que les trae la vida y de preparar a los estudiantes para un futuro cada vez más exigente, no siempre se obtienen los resultados esperados en los tiempos programados, ya que los ritmos de aprendizaje están marcados también por otros factores externos a esta, como lo son su familia (y más aún en la parte rural).

Es muy complicado lograr que un estudiante adquiera ciertos hábitos, como la lectura, la oralidad o la escritura, cuando en sus casas escasamente sus familiares han tenido hasta segundo de primaria y muy poco o nada se ve reflejado el hábito de estas habilidades comunicativas; no se quiere juzgar en ningún momento o culpar a los padres de familia; solo se pretende ser más objetivos al momento de evaluar a los estudiantes y ponerse en sus zapatos, además, los niños antes de salir de sus casas o cuando salen de la Institución deben ayudar con algunos trabajos del hogar. A partir del análisis de estas situaciones, surgió la siguiente pregunta de investigación:¿Cómo la aplicación de una unidad didáctica, basada en historia local promueve las competencias comunicativas en los estudiantes?

La metodología que se empleó es de enfoque cualitativo, bajo el paradigma socio-crítico y el diseño de Investigación Acción. Este proyecto se desarrolló en cuatro fases, las cuales apuntan a que los estudiantes mejoren sus prácticas comunicativas en el reconocimiento de su ambiente socio-cultural y lo puedan relacionar con la construcción del significado, en el que logren entender
Cuando comienza a fallar la comunicación, empiezan a debilitarse también los procesos de interacción con los demás. 


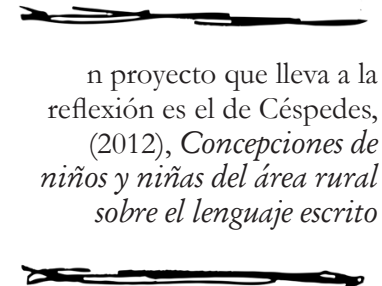

la forma de organizar sus ideas y darlas a conocer, haciéndose parte viva de la comunidad, conociendo su propia historia, sintiéndose orgullosos del legado literario, cultural y artístico, valorándolo, compartiéndolo con otros, enriqueciéndolo y recreándolo.

\section{1-Antecedentes}

Las competencias comunicativas, como eje fundamental en la educación y en la vida de todo individuo, son parte importante en su desarrollo personal y social. Estas competencias comunicativas constituyen diversos proyectos de investigación, que permiten superar los inconvenientes que se presentan a lo largo del período escolar. En la revisión documental y de antecedentes se encontraron referentes importantes que permiten tener claridad a esta propuesta didáctica.

En los antecedentes está el trabajo de Arboleda(2012), propuesta didáctica para mejorar la calidad de los discursos orales formales en estudiantes de quinto grado de educación básica primaria con y sin el apoyo del programa de audio digital Audacity, la cual busca que los estudiantes pasen de un discurso con poca fluidez verbal a un discurso oral formal; es decir, con lenguaje acorde al contexto, teniendo en cuenta nuevos usos lingüísticos, la coherencia y cohesión de las ideas, apoyados en las estrategias del programa, el cual es una herramienta para la producción de los discursos orales formales.

Otro trabajo de oralidad es el de Rincón y Rivera (2015), Fortalecimiento de la expresión oral en un contexto de interacción comunicativa, en el que se muestra, cómo, a partir de un diseño de estrategias comunicativas llamadas bioclases (espacios dinámicos en los cuales interactúa profesor y estudiante), se construye un aprendizaje significativo en un contexto en el cual se resalta la importancia de la radio como estrategia de aprendizaje, la cual se constituye como herramienta fundamental en el proceso pedagógico.

Lo avances que se dan en las diferentes propuestas no solo deben arrojar resultados positivos en la escuela, sino que han de tener eco en su entorno. Castro (2016), en Memoria social y narrativas audiovisuales en niños de Usme rural, muestra el trabajo con estudiantes de primaria en el que analiza el proceso de producción de narrativas audiovisuales. Allí, se videncia la construcción de la memoria social, un trabajo realizado con la comunidad, en el que se refleja la importancia de conservar todas esas tradiciones, memorias, relatos, costumbres y busca que los estudiantes, desde temprana edad, expresen espontáneamente la forma de pensar, que sean críticos, analíticos y participativos.

Un proyecto que lleva a la reflexión es el de Céspedes, (2012), Concepciones de niños y niñas del área rural sobre el lenguaje escrito, en el que los niños cuentan cómo el proceso de escritura se da como función pedagógica, es decir, para realizar tareas, cumplir con actividades o para aprobar un grado, no se da en situaciones reales de comunicación y no hay intervención por parte de los estudiantes para proponer 
actividades. La escuela rural cuenta con un cúmulo de saberes, tradiciones y cultura que permanecen vivos en el recuerdo de los habitantes y que están abiertas para ser contadas y plasmadas por las tiernas manos de quienes las escuchan.

La propuesta de Sigcha (2010), Elaboración y aplicación de un manual de ejercicios para desarrollar el lenguaje oral en los niños de 5-6 años de la escuela de práctica docente Agustín Albán 20092010 surge de la deficiencia que tienen los estudiantes al comunicarse y hacerse entender, ya que ellos mezclan fonemas porque los padres son pertenecientes a la familia lingüística Quichua del Ecuador. El manual de ejercicios recoge actividades que, poco a poco, incrementan su complejidad fomentado en el trabajo en equipo, ambientes escolares y el medio que rodea a los niños. Estas propuestas son fundamentales en cuanto las acciones llevadas a cabo por los docentes permiten promover las competencias comunicativas, y posibilitar otras prácticas pedagógicas. Del mismo modo, la unidad didáctica desarrollada en esta propuesta, permite fortalecer las competencias de oralidad y escritura tomando la historia local como propiciadora de aprendizajesignificativo y permitiendo que este sea conocido por otras personas, generando en los niños un sentido de compromiso y responsabilidad.

\section{2-Marco teórico}

\subsection{Competencias comunicativas}

El lenguaje expresado en todas sus formas, se constituye en un aporte vital para la humanidad, necesario e imprescindible en cualquier contexto en el que se desenvuelva el ser humano. Por ende, es el área de humanidades la encargada de formar, fortalecer y brindar acciones que permitan a los estudiantes ser competentes en cualquier acto comunicativo, en los Estándares Básicos de Competencias (2006):

La pedagogía de la lengua castellana centra su foco de atención e interés en el desarrollo de la competencia comunicativa de los estudiantes, en el sentido que estén en condiciones de identificar el contexto de comunicación en el que se encuentran $y$, en consecuencia, saber cuándo hablar, sobre qué, de qué manera hacerlo, cómo reconocer las intenciones que subyacen a todo discurso, cómo hacer evidentes los aspectos conflictivos de la comunicación, en fin, cómo actuar sobre el mundo e interactuar con los demás a partir de la lengua y, dese luego, del lenguaje (pp. 24-25).

De este modo, las competencias comunicativas permiten a los estudiantes formarse integralmente para que sean capaces de interpretar su propia realidad, expresarse libremente, participar y ejercer sus derechos como ciudadano, estas se van desarrollando y fortaleciendo en la escuela, quien es la encargada de posibilitar situaciones en las cuales los estudiantes interactúan y participan.

\subsection{Modelo educativos escuela nueva, en contextos rurales}

El programa Escuela Nueva que se viene trabajando en Colombia desde
La propuesta de Sigcha (2010), Elaboración y aplicación de un manual de ejercicios para desarrollar el lenguaje oral en los niños de 5-6 años de la escuela de práctica docente Agustín Albán 2009-2010 


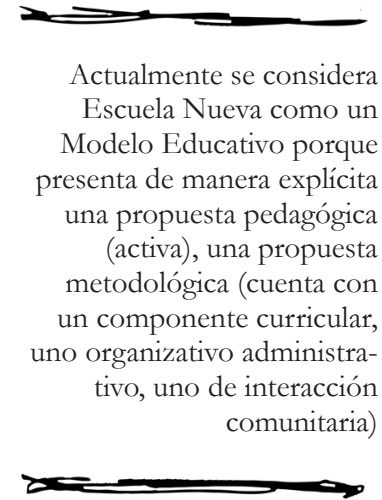

hace aproximadamente 35 años $y$ específicamente en las zonas rurales, se ha convertido en un modelo educativo que se articula con los PEI de las Instituciones. Para el Ministerio de Educación Nacional (MEN, 2010):

Actualmente se considera Escuela Nueva como un Modelo Educativo porque presenta de manera explícita una propuesta pedagógica (activa), una propuesta metodológica (cuenta con un componente curricular, uno organizativo administrativo, uno de interacción comunitaria) y una propuesta didáctica (cartillas, con unidades y guías, las cuales desarrollan una secuencia didáctica, (p.9).

Todos estos componentes, sumados al contexto en el cual se da este proceso de enseñanza-aprendizaje, como son los espacios, el ambiente natural, el material didáctico y la familia, constituyen el escenario apropiado para aplicar diversas estrategias metodológicas, con el fin de potenciar las competencias comunicativas.

Los niños y las niñas llegan a la escuela con un sinnúmero de conocimientos, habilidades y actitudes, es decir competencias, por lo que cobra importancia que los maestros y maestras observen cuidadosamente lo que los niños saben y saben hacer frente a diferentes situaciones. A partir de este reconocimiento el docente puede contextualizar las actividades pedagógicas propuestas por estas orientaciones.

De este modo, es necesario destacar las particularidades del entorno para plantear estrategias que signifiquen y le permitan interactuar al estudiante, de tal manera que sus avances sean fundamentales en la vida escolar y social. Además, el permanente contacto de los niños con la naturaleza les permite leer, dialogar y jugar con ella; ampliar el mundo real, al igual que construir e imaginar otros mundos fantásticos donde podrán recrear el libre universo de sus sueños, para traducirlo en palabras habladas o escritas.

\subsection{Memoria Social}

Decía el poeta bogotano José Asunción Silva (1968), "con el recuerdo bello de las cosas que embellecen el tiempo y la distancia, retornan a las almas cariñosas cual bandadas de blancas mariposas, los plácidos recuerdos de la infancia" (p.19). Los recuerdos, las experiencias vividas positivas o negativas, los conocimientos adquiridos, se convierten muchas veces en la mejor compañía y permiten alegrar momentos o traer al presente todo ese cúmulo de saberes que tienen los seres humanos en el baúl de la memoria, y que cobran un gran valor al momento de ser compartidos. Para Le Goff (1991), "las sociedades en las cuales la memoria social es principalmente oral o las que están constituyéndose una memoria colectiva escrita permiten entender mejor esta lucha por el dominio del recuerdo y de la tradición" (pp. 181-182). Es precisamente la memoria la que se pretende rescatar en esta interacción con la comunidad con el fin de promover las competencias orales y escritas, pues, para Ricoeur (2003) "no tenemos otro recurso, sobre la referencia al pasado, que la memoria 
misma" (p.40). La cual permite recorrer espacios y tiempo, dejando fluir las palabras para que quienes las escuchan sean artífices del propio conocimiento.

En este rescate de la memoria, son las competencias comunicativas el motor que mueve los procesos que permiten ampliar los horizontes de los estudiantes ya que, según Parra (1996), "la adquisición y la transmisión de capital cultural está en una íntima relación con el lenguaje. El lenguaje es también capital cultural y el aprendido en la escuela se convierte en un elemento que vincula al niño a la sociedad" (p.116). De este modo, lenguaje y memoria social rural son el complemento que promueve el desarrollo de las competencias comunicativas dentro y fuera del aula de clases, contando siempre con el apoyo de los padres de familia.

\subsection{Lectura}

Es una de las competencias a la que se le delega mayor responsabilidad a los docentes de primaria, quienes son los encargados de las grafías, ortografía, signos de puntuación y los demás aspectos que enriquecen el buen ejercicio de la lecto-escritura. Esta labor es más de los primeros años de edad escolar, y de ahí parte la necesidad de incrementar estrategias que permitan un mejor nivel en estos procesos. Para Díaz y Hernández (2010), “Cuando se lee un texto se realizan procesos complejos de construcción de significados y de atribución de sentidos, a partir de la información que éste provee” (p.7). Los estudiantes estructuran y organizan la información de acuerdo con los saberes previos, según la habilidad que tiene para relacionar las ideas, lo mismo que la capacidad que posee para interpretar y poner en práctica las diferentes estrategias que le permiten tener éxito en la comprensión textual, ya que la lectura es:

Un diálogo entre lo que dice el autor en el texto y lo que el lector construye en su mente. Si el lector no tiene saberes previos sobre lo que dice el texto y un conjunto de estrategias mentales para activar mientras lee, no entenderá el texto o lo hará parcialmente. (Sánchez, 2014, p.14).

Es importante integrar todos estos componentes para que exista un verdadero proceso de comprensión. Estos actos de lecto-escritura siempre se deben dar como procesos de construcción en contextos de comunicación y como prácticas en las cuales los estudiantes se involucren para edificar conocimiento, de ahí la importancia que los niños se apropien de estas habilidades no solo para realizar determinadas tareas escolares, sino que sea vivencial en el contexto, para exigir los derechos, reclamar y dar a conocer sus puntos de vista sobre un determinado tema, ya que:

El desafío que hoy enfrenta la escuela es el de incorporar a todos los alumnos a la cultura de lo escrito, es el de lograr que todos sus exalumnos lleguen a ser miembros plenos de la comunidad de lectores y escritores. (Lerner, 2001, p.25).

Los estudiantes no solo han de estar motivados para desarrollar las distintas actividades propuestas en el aula, sino que son también, el reflejo de las
El desafío que hoy enfrenta la escuela es el de incorporar a todos los alumnos a la cultura de lo escrito, es el de lograr que todos sus exalumnos lleguen a ser miembros plenos de la comunidad de lectores y escritores. 
prácticas diarias de la actividad docente, no se concibe, como se le puede exigir a un niño que escriba, lea, hable y escuche cuando el maestro no hace real ejercicio de estas competencias comunicativas. Sánchez (2014) dice, con respecto a la lectura, que: "Es uno de los principales medios a través de los cuales niños, niñas y jóvenes aprenden nuevos conocimientos, confrontan sus saberes previos y modifican sus ideas sobre la realidad del mundo" (p. 25). Una vez que los estudiantes han adquirido el hábito de la lectura, no les bastará solo con lo que el docente desarrolle en clase, sino que la curiosidad por conocer sobre determinados temas provocará un deseo personal por ahondar en los significados, esta tarea debe ser asumida no solo por los docentes de lenguaje, sino que es un trabajo en conjunto con los maestros de todas las asignaturas y de todos los grados (Quitián, 2017).

\subsection{Escritura}

La competencia comunicativa escritora es una herramienta que facilita y mejora las relaciones de los seres humanos, cada persona le impregna su estilo particular, muchas veces los adultos no han descubierto en ellos mismos, que tienen una riqueza inmensa para contar, para hablar sobre determinados temas como la poesía, política, cuentos, en fin, con mayor razón los niños no son conscientes de sus capacidades, por eso el rol del docente que descubre y potencializa esta habilidad; es necesario, al momento de crear un texto, hacerle ver al estudiante que sus escritos deben responder a unas cuestionamientos básicos, como: ¿para quién se escribe?, ¿por qué se escribe?, ¿cómo se escribe?, de esta manera el escritor tiene en su mente quienes serán sus posibles lectores (Castaño, 2014).

De este modo, el estudiante no construye su propio conocimiento, puesto que sus prácticas de escritura son impuestas o determinadas bajo ciertos parámetros que, en ocasiones, poco aportan al aprendizaje significativo. Bustamante y Jurado (1997), con respecto a las prácticas de aula, afirman que: "el escrito tal como se ha abordado en la escuela colombiana, no ha intervenido realmente en los procesos de comprensión y de producción escrita, es decir, no ha logrado establecer cambios verdaderamente significativos y complejos a nivel cognitivo" (p.102). Por lo que es importante plantear estrategias que promuevan la interacción del estudiante con sus textos, que en su mente siempre este también la posición de lector, y al escribir un determinado texto; y que este, cumpla con su objetivo, cualquiera que sea, describir, narrar, informar o recrear. Los esquemas mentales que se hacen acerca de lo que se va escribir, el pensar en el otro como lector que interactúa con el escrito, determinan en gran proporción la calidad del texto. Por lo tanto:

La lengua escrita no puede perder sus grandes fines: de organización cognitiva, comunicativos, estéticos. En definitiva: debe permitir nombrar la realidad y transformarla. En el momento histórico oportuno-el siglo XXI, la edad de la información- de consolidar una competencia escritural y lectora plena en que nuestros niños y niñas adquieran 
eficientes competencias comunicativas de comprensión lectora y de producción textual (Negret, 2005, p.11).

No obstante, en educación primaria son muchos los programas que implementa el Ministerio de Educación Nacional para mejorar y superar las dificultades que presentanlos estudiantes, programas como: Todos a Aprender, Las letras van por Colombia, Colección semilla, Cartillas que traen todos los documentos de referencia como son los Derechos Básicos de Aprendizaje, Estándares de Calidad, Mallas de Aprendizaje, Orientaciones Pedagógicas, entre otros; los cuales recalcan y hacen visible lo imprescindible que es el manejo de las competencias comunicativas.

\subsection{Oralidad}

Hablar fue una de las primeras formas de comunicación en desarrollarse. A través de la expresión oral, se rescata la historia, se pueden hacer investigaciones y se conserva la cultura. Ong (1987) destaca la importancia de la oralidad, ya que "la expresión oral es capaz de existir sin ninguna escritura en absoluto; empero nunca ha habido escritura sin oralidad" (p. 18). Por lo tanto, se podría decir que las habilidades como la lectura y la escritura no pueden existir independientemente de la oralidad y escucha, puesto que estas se desprenden del lenguaje hablado.

Los seres humanos aprenden a hablar en sus hogares, pero es la escuela la que debe edificar sobre los cimientos que traen los estudiantes,

El objetivo fundamental consiste en capacitar a los niños en la producción y comprensión de discursos en su lengua materna, sobre el conocimiento del mundo natural, las experiencias de la vida cotidiana, la interacción social, y la participación en la construcción de la nueva sociedad civil, más digna y más humana que la presente.(Álvarez y Cortés, 1990, p.15)

En el lenguaje oral, las dificultades que más presentan los estudiantes son: la timidez, poca fluidez verbal, temor a equivocarse y que se burlen de ellos, entre otras. Se cree que como los niños aprenden a hablar en sus hogares, por ende ya tienen esta habilidad aprehendida. Según Parra (1996), "sí, es imprescindible, que el maestro fomente y estimule la espontaneidad expresiva del niño, que posea un manejo hábil del lenguaje, que procure conocer las características y las particularidades de la comunidad" (p.18). Por lo tanto, es labor del maestro propiciar estrategias que les permita a los estudiantes superar sus debilidades.

Fausto Sandoval (citado en López y Jung, (1998) comenta: "soy de aquellos que quieren matrimoniar lo oral con lo escrito en mi cultura. La oralidad es una doncella que ha marchado sola por mucho tiempo, aunque acechada constantemente por un pretendiente poderoso que podríamos identificar como lo escrito" (p.81). Así como la competencia escritora permite dar cuenta de los testimonios, relatos o anécdotas, tiene tanta importancia como la oralidad, ya que las dos son fundamentales para que cualquier individuo se pueda desenvolver y surgir en un medio socio-
En el lenguaje oral, las dificultades que más presentan los estudiantes son: la timidez, poca fluidez verbal, temor a equivocarse y que se burlen de ellos, entre otras. 
cultural que, día a día, exige más.

Es necesario recurrir a estrategias que promuevan en los estudiantes actos como dialogar, criticar constructivamente, opinar, expresarse libremente, que escuchen y sean escuchados, que se reconozcan como agentes activos dentro de su comunidad; cuando estos procesos se logren se podría decir que la escuela está siendo posibilitadora de artesanos de la palabra escrita y oral; por tal razón, los recursos con los que cuenta la Institución, como las TIC y los medios de comunicación como las emisoras, son valiosos. Para Río(s.f.), "Muchos centros escolares han descubierto que la educación es básicamente comunicación y que los alumnos pertenecen a una sociedad nutrida, fundamentalmente de mensajes y que, por tanto, el uso de la radio presenta sobre otros medios muchas más ventajas." (p.26). De este modo, la radio, es una herramienta importante con la cual se trasciende a otros lugares, llega a muchas personas y permite que las prácticas de aula atraviesen fronteras.

\subsection{Escucha}

El acto de escuchar está estrechamente relacionado con el de hablar. Para que exista una verdadera comunicación, es importante que estas dos habilidades se den de manera simultánea, puesto que:

Tiene que ver con elementos pragmáticos como el reconocimiento del contexto social, cultural, ideológico desde el cual se habla; además está asociado a complejos procesos cognitivos ya que a diferencia del acto de leer en el que se cuenta con el impreso como soporte de la significación, escuchar implica ir tejiendo el significado de manera inmediata. (Lineamientos Curriculares, 1998, p.50).

Cuando se presenta una conferencia en un lugar determinado, no todas las personas que allí asisten reciben el mensaje o lo perciben del mismo modo. Para Ong (1994), "No todos los integrantes de un público grande entiende cada palabra pronunciada por un hablante, aunque esto sólo se deba a problemas acústicos. Es conveniente que el orador diga lo mismo, o algo equivalente, dos o tres veces" (p.46). Del mismo modo, cuando se presenta ante un público hay que ser reiterativo y, si es posible, ejemplarizar para que el mensaje quede claro. Por tal razón, en las aulas de clase hay que abrir los espacios y propiciar escenarios en los cuales los estudiantes se involucren, fomentando la expresión oral y la escucha, así, las entrevistas realizadas a la comunidad, posibilitan en los estudiantes habilidades y destrezas.

\section{Metodología}

Esta propuesta se desarrolla en el Paradigma Socio Critico, puesto que el investigador se constituye como un sujeto colectivo de autorreflexión que se involucra en el proyecto de manera directa, para transformar y generar un cambio social. El enfoque cualitativo concentra sus esfuerzos investigativos en la descripción, comprensión e interpretación de los significados que los estudiantes le dan a sus propias acciones. Para Suárez (2001), "la investigación cualitativa parte de una concepción de la realidad como totalidad que se construye en la interacción comunicativa de sus integrantes y que 
se debe estudiar teniendo diferentes teorías como referentes para abordar una realidad múltiple" (p.179). De este modo, los actores del proyecto interactúan con la comunidad y construyen su propio aprendizaje desde diferentes perspectivas metodológicas.

La propuesta de aula es investigaciónacción, ya que permite que los estudiantes se vinculen en el proceso e interactúen con la comunidad, dando paso a la construcción del conocimiento y reflexionando sobre sus prácticas.

La población objeto de investigación corresponde a dos estudiantes de cuarto y seis del grado quinto primaria, de la Sede Gaunza Abajo. Los estudiantes provienen de familias campesinas (seis de los estudiantes viven con familias constituidas por padre y madre, y dos viven solo con la madre). La mayoría de los padres de familia cursaron hasta tercero de primaria. Su principal fuente de ingresos es la agricultura y la ganadería a pequeña escala, y algunas madres trabajan en casas de familia.

Para la recolección de datos, se realizaron entrevistas a la comunidad las cuales fueron grabadas en video, audios de pruebas diagnósticas y finales, escritos como pruebas de entrada y también de las diez entrevistas de los estudiantes, entrevista de inicio y final, mediante la aplicación de la propuesta se han tenido en cuenta aspectos como: coherencia y cohesión del texto, uso de vocabulario desconocido, longitud, expresión corporal, entre otros.

\section{Procedimiento}

El desarrollo de este proyecto se ha dividido en cuatro fases:

En la primera, se diagnostica el nivel de desempeño de los estudiantes en las competencias comunicativas. En cuanto a escritura, esta se mide a través de pruebas internas, tales como: pedir inicialmente a los estudiantes que escriban sobre su forma de vida, la evaluación de esta actividad se hizo mediante una rejilla que lleva aspectos como: extensión del texto, uso de vocabulario desconocido, cantidad de palabras, coherencia y cohesión; en cuanto a la oralidad, se les pidió a los estudiantes que hablen sobre las mascotas, ya que es un tema en común y del agrado de todos, se les graba; luego se valora, también, mediante una rejilla que tiene aspectos, como: tiempo utilizado al momento de intervenir, número de palabras, tono y timbre de la voz, dominio de público y uso de palabras diferentes al vocabulario empleado usualmente.

La segunda fase fue el diseño de la unidad didáctica para promover las competencias lingüísticas, para estas actividades se estructuran once talleres: La actividad 1, es La entrevista, con el fin de que los estudiantes se apropien del rol que van a desempeñar y conozcan más a fondo lo concerniente a este tema, actividad 2, signos de puntuación y reglas ortográficas, se relaciona con la entrevista sobre el ordeño; 3, el texto narrativo con la entrevista sobre los amasijos; 4, texto descriptivo, conjunciones, con la entrevista del proceso del fique; 5 , el párrafo y conectores de temporalidad 
con la entrevista de envueltos al horno; 6 , los adjetivos, con la entrevista de los tejidos en crochet; 7 , las preposiciones, con la entrevista sobre la molienda, 8 , regionalismos, con la entrevista sobre la agricultura, 9, la comunicación, con la entrevista sobre el cuidado y cría de ovejas; 10, tradición oral, con la entrevista sobre el proceso del café, 11, tiempos verbales, con la entrevista sobre ganadería y cuidado de cerdos.

Basados en el modelo pedagógico de la Institución Enseñanza para la Comprensión, los talleres contienen los Estándares Básicos de Calidad, los Derechos Básicos de Aprendizaje, tópicos generativos, hilos conductores, competencias, pragmática, semántica y sintáctica, la evaluación diagnóstica formativa y los tres momentos de cada una de las clases, como son: la exploración que se relaciona con los saberes previos del estudiante, la estructuración, la cual está dada mediante la práctica y apropiación de los conceptos; y la transferencia, que se relaciona con el reconocimiento, comprensión y valoración de cada uno de los conceptos dados.

Se plantea para cada uno de los talleres, realizar una entrevista a diferentes miembros de la comunidad con el fin de conocer sobre las labores del campo. Una vez recopilada esta información, los estudiantes se darán a la tarea de escribir sobre lo observado en cada salida. Los talleres se estructuran de tal manera con el fin de que las competencias comunicativas de lectura, habla, escucha y escritura estén siempre presentes a través de todas las actividades, haciendo énfasis en la oralidad y la escritura.

La tercera fase consiste en ejecutar las entrevistas según el cronograma establecido. Previo a la entrevista, se aplica un taller que permite dar claridad al escrito que se va a realizar, aquí se evalúan los mismos aspectos de la prueba diagnóstica para la escritura. En cuanto a la oralidad se les pide a los estudiantes que hablen sobre los temas de cada una de las entrevistas y, del mismo modo, se evalúa con la rejilla de la prueba inicial.

La cuarta fase es socializar a través de los programas de radio Juventud Olayista y Sutatenza Stereo, las entrevistas y los escritos construidos por los estudiantes.

\section{Análisis de resultados}

La aplicación de la Unidad Didáctica evidenció un avance importante en las competencias escritora y oral, ya que los estudiantes redactan textos más extensos hilándolos con coherencia, cohesión y fluidez, puesto que tienen argumentos suficientes para contar. Aún falta hacer uso de reglas ortográficas, signos de puntuación y categorías gramaticales. En cuanto a la oralidad, se obtuvo un resultado significativo, puesto que los estudiantes lograron disminuir el temor de enfrentarse a un público y se motivaron a participar preguntando $\mathrm{y}$ contando las experiencias vividas.

El desarrollo de la propuesta motivó a los estudiantes, ya que permitió conocer más su contexto, interactuar con compañeros y comunidad, lo cual permite reafirmar las diferentes teorías planteadas por los autores, cuando 
hablan del aprendizaje significativo, de estrategias que potencialicen los conocimientos que traen de sus hogares, del conocimiento de la historia local como fuente importante de tradiciones culturales que permiten a los estudiantes poner en práctica las competencias escritoras y orales, además de la radio como herramienta pedagógica para fortalecer estos procesos.

\section{Conclusiones}

Las competencias comunicativas se fortalecen y enriquecen en el interactuar diario, cuando la escuela es posibilitadora de experiencias significativas, donde el estudiante construye su propio conocimiento, los resultados no solo se evidencian en lo académico sino en el transcurso de la vida.

El contexto rural es un lugar propicio para el desarrollo de la propuesta; de esta manera, se evidencia cómo el rescate de la oralidad sobre las labores del campo permitieron afianzar los procesos escritor-oral, el rescate de la identidad, costumbres y trabajo en equipo.

El resultado de los escritos de los estudiantes fue un trabajo interesante, por tal razón se editó un libro con treinta y cuatro escritos, titulado "Relatos de Gaunza Abajo" motivando

aún más a los niños para continuar en este proceso, del que ya se tienen planeadas otras entrevistas.

\section{Referencias}

Álvarez, J., \& Cortés, M. (1990). Discurso y desarrollo de la competencia comunicativa en la educación básica. Bogotá, Colombia: Trilce Editores

Arboleda, A. (2012).Propuesta didáctica para mejorar la oralidad de los discursos orales formales de estudiantes de quinto grado de educación básica primaria con y sin el apoyo del programa de audio digital Audacity..Recuperado de: ayura.udea.edu.co:8080/jspui/bitstream/123456789/208/1PAO630.pdf.

Bustamante, G.,\& Jurado, F. (1997). Entre la lectura y la escritura. Santafé de Bogotá, Colombia: Magisterio.

Cañizález Mesa, N. E., \& Benavides Rozo, F. A. (2018). La didáctica como herramienta de la reflexión docente. Educación Y Territorio, 6(11), 105 - 145. Recuperado a partir de https://jdc.edu.co/revistas/index.php/reyte/article/ view/48

Castaño, A. (2014). Prácticas de lectura en el aula. Bogotá, Colombia: Ministerio de Educación Nacional.

Negret, J. (2005). La escritura antes de la escritura Historia del desarrollo individual del lenguaje escrito. Bogotá Colombia: Herramientas y Gestión, E.A.T.

Castro, N. (2016). Memoria social y narrativas audiovisuales en niños de Usme rural. Recuperado de repository.udistrital.edu.co/bitstream/11349/3095/1C

Las competencias

comunicativas se fortalecen y enriquecen en el interactuar diario, cuando la escuela es posibilitadora de experiencias significativas, donde el estudiante construye su propio conocimiento 
astroNelsonFederico2016.pdf

CÉSPEDES, L. (2012). Concepciones de niños y niñas del área rural sobre el lenguaje escrito Recuperado de repositorio.utc.edu.co/dspace/bitstream/ handle11059/2821/372634C422.pdf;jsessionid=CEO51COF79398D8A421 CF856E9217DD5? sequence $=$

Díaz, F., \& Hernández, G. (2010). Leer y escribir para un aprendizaje significativo y reflexivo. México: McGraw Hill.

LERNER, D. (2001). Leer y escribir en la escuela: lo real, lo posible y lo necesario. México: Fondo de Cultura Económica.

LE GofF, J. (1991). El orden de la memoria. El tiempo como imaginario. Barcelona, España: Paidós.

LÓPez, L,. \& Jung, I. (1998). Sobre las huellas de la voz. Madrid, España: Morata, S.L.

Ministerio de Educación Nacional. (1998). Lineamientos Curriculares Lengua Castellana. Bogotá Colombia: Nomos Impresores S.A.

Ministerio de Educación Nacional. (2006). Estándares Básicos de Competencias en Lenguaje, Matemáticas, Ciencias y Ciudadanas. Bogotá, Colombia: Imprenta Nacional de Colombia.

Ministerio de Educación Nacional. (2010). Escuela Nueva Manual de Implementación Escuela Nueva Generalidades y Orientaciones Pedagógicas para Transición y Primer Grado. Tomo 1. Recuperado de: www.mineducación. gov.co/1759/articles-340089_archivopdf.orientaciones_pedagogicas_tomo1. pdf

Ong, W. (1987). Oralidad y escritura tecnologías de la palabra. México: Fondo de Cultura Económica.

Parra, R. (1996). Escuela y Modernidad en Colombia. La Escuela Rural. Colombia: Tercer Mundo Editores.

Ricoeur, P. (2003). La memoria, la historia, el olvido. España: Trotta.

Río, P. (s.f.). La radio en el Diseño Curricular. Madrid, España: Bruño.

Rincón, Y.. \& PARRA, A. (2015). Fortalecimiento de la expresión oral en un contexto de interacción comunicativa. Recuperado de repositorio.uptc.edu.co/jspui/ bitstream/001/1513/1TGT-149.pdf

Sánchez, C. (2014). Prácticas de lectura en el aula. Bogotá, Colombia: Ministerio de Educación Nacional.

Sigcha, M. (2010). Elaboración y aplicación de un manual de ejercicios para desarrollar el lenguaje oral en los niños de 5-6 años de la escuela de práctica docente Agustín Albán 2009-2010. Recuperado de repositorio.utc.edu.ec/ bistream/27000/279/1/T-UTC-0278.pdf 